\title{
Stable coupled conjugate solitary waves in optical fibers
}

\author{
S. Wabnitz \\ Laboratoire de Physique, Université de Bourgogne, 9 Avenue A. Savary, B.P. 400, 21011 Dijon, France \\ J. M. Soto-Crespo \\ Instituto de Optica, Consejo Superior de Investigaciones Cientificas, Serrano 121, 28006 Madrid, Spain
}

Received October 6, 1997

Four-wave mixing of an intense continuous-wave pump beam with an ultrashort soliton signal in an optical fiber is theoretically analyzed. A novel class of stable two-color coupled solitary waves is found. These vector parametric solitons represent the optimal frequency conversion of an ultrashort pulse. () 1998 Optical Society of America

OCIS codes: $\quad 300.2570,350.7420,060.2280$.

As is well known, optical phase conjugation undoes optical signal distortions on propagation in dispersive and nonlinear media. ${ }^{1}$ Generation of the conjugate replica of a signal at the middle of a fiber-optic transmission link can compensate for dispersive and nonlinear broadening. ${ }^{2}$ When it is applied to soliton systems, mid-span spectral inversion also removes other important transmission capacity-limiting effects such as pulse interactions. ${ }^{3}$ Four-wave mixing in fibers can also be exploited for wavelength shifting and time demultiplexing of high-bit-rate signals. For efficient and broadband frequency conversion, pumping near the zero-dispersion wavelength of a dispersion-shifted fiber is required. ${ }^{4,5}$ As a result, the fiber group-velocity dispersion (GVD) has opposite signs at the signal and conjugate wavelengths. In the conjugation of ultrashort solitons, that is, for dispersion-shifted fiber lengths equal to or longer than the dispersion distance, the effect of finite GVD at the signal and conjugate wavelengths sets a fundamental limit on the conversion efficiency.

Cross-phase modulation between solitons at different wavelengths leads to coupling between dark and bright solitons in opposite dispersion regimes. ${ }^{6}$ In this Letter we analyze the parametric coupling of a cw pump with short signal and conjugate pulses. The purpose of this study is the search for stable twocomponent solitary waves that permit the propagation of a solitonlike pulse at the conjugate frequency, owing to parametric coupling with a pulse at the signal frequency. As we shall see, in a lossy fiber two parametrically coupled bright solitons can be generated, irrespective of the sign of the signal GVD, and correspond to the optimal frequency conversion of ultrashort pulses.

Three-wave mixing of a pump $E_{p}$, a signal $E_{s}$, and a conjugate wave $E_{c}$ in optical fibers is described by the coupled nonlinear Schrödinger equations ${ }^{7}$

$$
\begin{aligned}
& i \frac{\partial E_{p}}{\partial z}+\frac{i}{V_{p}} \frac{\partial E_{p}}{\partial t}- \frac{\beta_{2 p}}{2} \frac{\partial^{2} E_{p}}{\partial t^{2}}+i \Gamma_{p} E_{p} \\
&+R\left[\left|E_{p}\right|^{2}+2\left(\left|E_{s}\right|^{2}+\left|E_{c}\right|^{2}\right)\right] E_{p} \\
&+2 R E_{s} E_{c} E_{p}{ }^{*} \exp (i \delta k z)=0, \\
& 0146-9592 / 98 / 040265-03 \$ 10.00 / 0
\end{aligned}
$$

$$
\begin{gathered}
i \frac{\partial E_{s}}{\partial z}+\frac{i}{V_{s}} \frac{\partial E_{s}}{\partial t}-\frac{\beta_{2 s}}{2} \frac{\partial^{2} E_{s}}{\partial t^{2}}+i \Gamma_{s} E_{s} \\
+R\left[\left|E_{s}\right|^{2}+2\left(\left|E_{c}\right|^{2}+\left|E_{p}\right|^{2}\right)\right] E_{s} \\
+R E_{p}{ }^{2} E_{c}{ }^{*} \exp (-i \delta k z)=0 \\
i \frac{\partial E_{c}}{\partial z}+\frac{i}{V_{c}} \frac{\partial E_{c}}{\partial t}-\frac{\beta_{2 c}}{2} \frac{\partial^{2} E_{c}}{\partial t^{2}}+i \Gamma_{c} E_{c} \\
+R\left[\left|E_{c}\right|^{2}+2\left(\left|E_{s}\right|^{2}+\left|E_{p}\right|^{2}\right)\right] E_{c} \\
+R E_{p}{ }^{2} E_{s}{ }^{*} \exp (-i \delta k z)=0 .
\end{gathered}
$$

Here $\Gamma_{p} \simeq \Gamma_{s} \simeq \Gamma_{c} \equiv \Gamma$ is the fiber loss coefficient, $R_{p}=2 \pi n_{2} /\left(\lambda_{p} A_{\text {eff }}\right) \simeq R_{s} \simeq R_{c} \equiv R, n_{2}$ is the nonlinear refractive index, and $A_{\text {eff }}$ is the fiber core's effective area. Moreover, $\delta k=k_{s}+k_{c}-2 k_{p}=\beta_{2 p} \Omega^{2}$ is the linear propagation constant mismatch, $V_{j}$ and $\beta_{2 j}$ are the group velocities and chromatic dispersions, respectively, at $\lambda_{j}(j=p, s, c)$, and $\Omega=\left|\omega_{p}-\omega_{s}\right|$. Let us consider the frequency conversion of a solitonlike pulse in the presence of a strong cw pump; i.e., we take $\left|E_{s}\right|,\left|E_{c}\right| \ll\left|E_{p}\right|$ and neglect pump depletion. These conditions are typically met in the case of intracavity frequency conversion, in which soliton pulses recirculate in a fiber loop of length $L$ and a short section of cw-pumped fiber (of length $L_{p}$ ) provides parametric gain. ${ }^{8}$ Moreover, for $\lambda_{p}$ close to the zero-dispersion wavelength, $V_{s} \simeq V_{c} \equiv V_{g}$, whereas $\beta_{2 s}=-\beta_{2 c} \equiv \beta$; with no restriction on the generality of our treatment, we can take $\beta_{2 s}<0$. Under the above simplifying conditions, we average Eqs. (1) over a circulation inside the fiber loop and obtain dimensionless coupled nonlinear Schrödinger equations for the signal and conjugate pulses:

$$
\begin{aligned}
i \frac{\partial u}{\partial Z}+\frac{1}{2} \frac{\partial^{2} u}{\partial T^{2}}+i \alpha u+i \gamma v & -\rho u \\
-\sigma\left(|u|^{2}+2|v|^{2}\right) u & =0, \\
i \frac{\partial v}{\partial Z}+\frac{1}{2} \frac{\partial^{2} v}{\partial T^{2}}+i \alpha v+i \gamma u & -\rho v \\
+\sigma\left(|v|^{2}+2|u|^{2}\right) v & =0,
\end{aligned}
$$

where $Z=z / z_{0} \equiv z|\beta| / t_{0}{ }^{2}, t_{0}$ is an arbitrary time unit, $T=\left[t-\left(z / V_{g}\right)\right] / t_{0},(u, v)=\left[E_{s} \exp (i \Delta k z / 2)\right.$,

(C) 1998 Optical Society of America 
$\left.-i E_{c}{ }^{*} \exp (-i \Delta k z / 2)\right] \sqrt{R t_{0}{ }^{2} /|\beta|}$, and $\sigma=-1$. Moreover, $\Delta k=\delta k-2 R P \eta$ is the total wavevector mismatch, $P \equiv\left|E_{p}\right|^{2}$ is the cw pump power, and $\eta=L_{p} / L \leq 1$. In Eqs. (2) the loss coefficient is $\alpha \equiv \Gamma z_{0}$, the parametric coupling coefficient reads as $\gamma \equiv \eta R P z_{0}$, and $\rho \equiv-(\delta k / 2+\eta R P) z_{0}$.

We intend to study here the existence and stability of coupled solitary-pulse solutions of Eqs. (2). Next, we investigate the possibility of generating stable two-component waves by injecting a signal pulse into the fiber. The vector solitons thus represent an optimal solution for the phase conjugation of a stream of ultrashort pulses. Before we consider the pulselike solutions of Eqs. (2), a simple analysis permits us to identify the conditions for stability of the low-power background. We can find the conditions for damping the continuum in Eqs. (2) (neglecting at first the presence of solitons as source of radiation) by considering a cw solution in the form $\left(u_{r}, v_{r}\right)=(A, B) \exp (i \lambda z)$. Neglecting nonlinear terms and time derivatives, we find that the conditions $\alpha>0$ and $\alpha^{2}>\gamma^{2}-\rho^{2}$ are necessary for the suppression of the cw growth.

Let us consider now the coupled bright solitary waves of Eqs. (2). Figure 1 shows the evolution with distance $Z$ of the amplitudes of a two-component stationary solution of the type

$u(Z, T)=U(T) \exp (-i q Z), v(Z, T)=V(T) \exp (-i q Z)$,

where $q$ is a real constant. We found the two complex amplitudes $U(T)$ and $V(T)$ and wave vector $q$ of the coupled pulselike stationary solutions numerically by inserting ansatz (3) into Eqs. (2) and by applying a shooting procedure to the resulting set of second-order ordinary differential equations. In Fig. 1 the stationary solution (with $q=0.26$ ) was used as the initial condition for Eqs. (2), which was then integrated along distance $Z$ by a standard split-step beam propagation code. In Fig. 1 the linear loss $\alpha=0.229$ exactly balances the parametric gain $\gamma=0.4$, whereas the wavevector mismatch $\rho=1$ compensates for the self- and cross-phase modulation phase shifts between the signal and conjugate pulses. As shown in Fig. 1, the numerical integration of Eqs. (2) clearly shows that the stationary solution is spatially unstable: Both pulse components decay at $\sim Z=30$. We investigated the linear stability of the two-component bright pulse solutions by considering the perturbed solutions

$$
\begin{aligned}
u(Z, T) & =[U(T)+f(Z, T)] \exp (-i q Z), v(Z, T) \\
& =[V(T)+g(Z, T)] \exp (-i q Z),
\end{aligned}
$$

where

$f(Z, T)=f_{0}(T) \exp (\delta Z), g(Z, T)=g_{0}(T) \exp (\delta Z)$.

By inserting Eq. (4) into Eqs. (2) and after linearizing the equations for perturbations $f$ and $g$ about a vector stationary wave solution, we obtained the profile of the most unstable perturbation eigenmode and its corresponding growth rate $\delta$ by means of a Crank-Nicholson procedure as described in detail else- where. ${ }^{9} \quad$ For example, for the case in Fig. 1 one finds the exponential growth rate coefficient $\delta=0.473$, in full agreement with numerical estimations obtained by integration of Eqs. (2) with different step sizes.

By slightly increasing the linear loss to $\alpha=0.277$ while keeping the other parameters fixed, we found that the numerical integration of Eqs. (2) with an initial condition given by the unstable stationary wave profile (in this case, $q=0.21$ ) with an additional small field proportional to the corresponding eigenperturbation evolved into a new stable coupled pulselike solution, as shown in Fig. 2. Note that the $V$ component of the vector soliton has a dip in its center and is temporally broader than its $U$ counterpart. This stable vector soliton represents a pair of signal and conjugate pulses of comparable amplitude and time width. Therefore, under such conditions, the signal pulse propagates along the fiber unchanged, along with a similar phase-conjugate replica. Clearly, each individual pulse of this coupled solitary pair is not in itself a soliton solution of the scalar nonlinear Schrödinger equation.

We numerically investigated (Fig. 3) the basin of attraction from the unstable into the stable coupled solitary waves as a function of both parametric gain $\gamma$ and loss $\alpha$ coefficients. As can be seen from Fig. 3, there is a relatively narrow stripe in the $(\gamma, \alpha)$ plane (the shaded area), which leads to decay from an unstable into a stable pulse pair. Above the boundary of existence of these asymptotically stable solutions, the unstable waves evolve into $Z$-periodic localized solutions, or breathers.
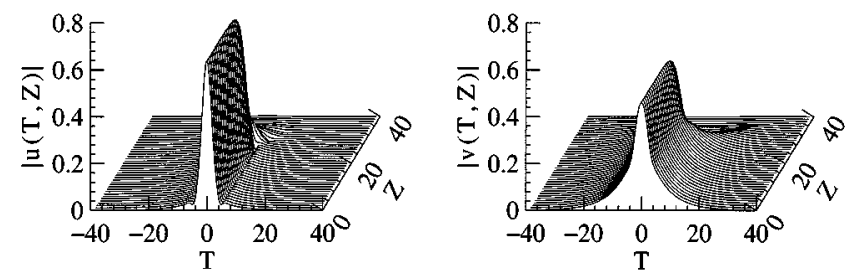

Fig. 1. Decay of unstable coupled signal and conjugate pulse amplitudes versus time $T$ and distance $Z$ for $\alpha=$ $0.229, \gamma=0.4$, and $\rho=-\sigma=1$.
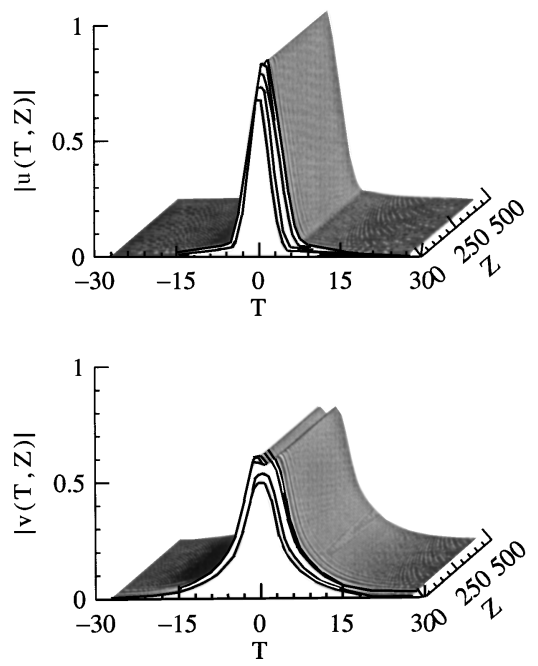

Fig. 2. Stable propagation of coupled signal and conjugate pulses as in Fig. 1 but with $\alpha=0.277$. 


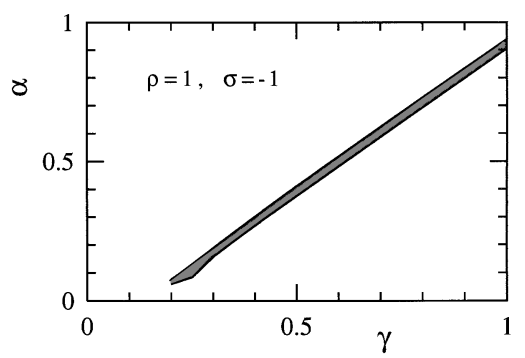

Fig. 3. Gray area, basin of attraction from unstable into stable conjugate solitary waves versus parametric gain $\gamma$ and $\operatorname{loss} \alpha$.
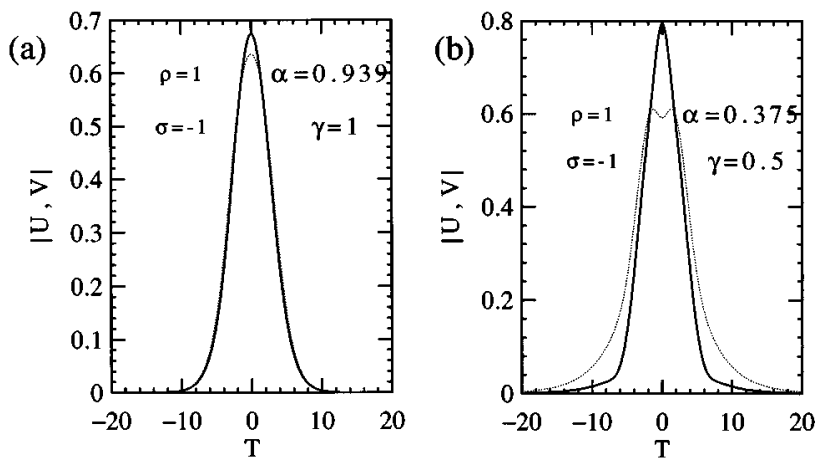

Fig. 4. Amplitude profiles [solid (dotted) curve for $U(V)$ ] of stable conjugate solitons.
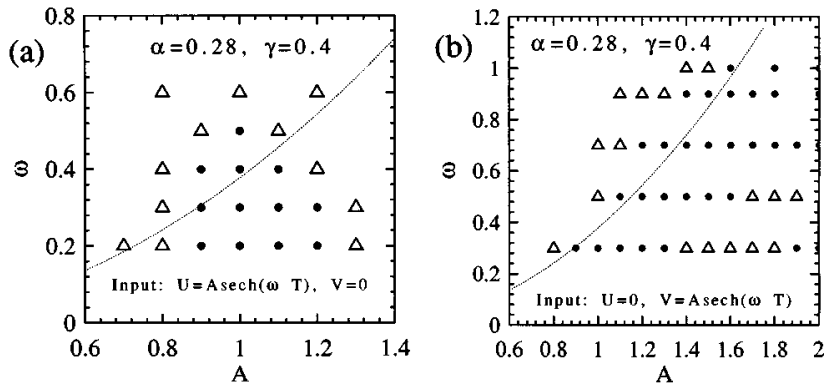

Fig. 5. Basin of attraction into stable coupled solitons versus initial amplitude $A$ and inverse width $\omega$ for an input signal in either (a) the anomalous or (b) the normal GVD regime.

The two components of the stable vector solitons have nearly the same hyperbolic secant profile at large values of $\alpha$ and $\gamma$; see, e.g., Fig. 4(a). This means that in the presence of strong dissipation and parametric gain one obtains coupled bright solitonlike pulses for both signal and idler, irrespective of the GVD sign. Indeed, it is known that in the normal GVD regime bright dissipative solitary waves can result from a balance between gain and loss. ${ }^{10}$ Reducing $\alpha$ below 0.5 broadens the $V$ component of the vector pulse in time and causes a dip at the center that corresponds with the peak of the $U$ pulse [Fig. 4(b)] with $\alpha=0.375$ and $\gamma=0.5$. As the loss is reduced further, the $V$ pulse (in the normal GVD) resembles a gray soliton upon a finite background. Hence, whenever GVD prevails over the dissipative terms, a pronounced temporal asymmetry between the two soliton components is observed.

So far we have considered the existence and stability of conjugate solitary waves in a fiber. From the viewpoint of applications, the main issue is the possibility of generating such solitary waves by means of a single pulse injected at the signal wavelength (along with the cw pump). We thus performed extensive numerical solutions of Eqs. (2) with an initial signal pulse of the form $u(Z=0, T)=A \operatorname{sech}(\omega T)$, and vanishing input idler $v(Z=0, T)=0$. The filled circles in Fig. 5(a) illustrate the basin of attraction from the above initial conditions into stable coupled solutions as a function of initial amplitude $A$ and inverse width $\omega$. The open triangles represent the initial conditions that led to decay of both pulses. The dotted curve in Fig. 5(a) illustrates the relation $2 A^{2} / \omega=E_{\mathrm{ss}}$, where $E_{\mathrm{ss}}$ is the energy corresponding to the stable stationary solution. Quite remarkably, Fig. 5(b) reveals that the basin of attraction into stable conjugate solitary waves is even broader when the input pulse is injected into the $v$ component, that is, for a signal in the normal GVD regime (and a conjugate pulse in the anomalous GVD). Indeed, the solitonic pulse compression mechanism inherent in the anomalous GVD regime enhances the reorganization of energy at the idler frequency into a narrow pulse. In real units, with $t_{0}=3.1 \mathrm{ps}, \beta=-1 \mathrm{ps}^{2} / \mathrm{km}$, fiber loss $0.24 \mathrm{~dB} / \mathrm{km}$, and $R=3.3(\mathrm{~W} / \mathrm{km})^{-1}$, we obtain $z_{0}=10 \mathrm{~km}$ and $\alpha=0.277$. Moreover, with $\eta=0.1$, the conditions $\gamma=0.4$ and $\rho=1$ are satisfied with pump power $P=120 \mathrm{~mW}, \beta_{2 p}=-0.2 \mathrm{ps}^{2} / \mathrm{km}$, and a pump-signal wavelength spacing of $1.8 \mathrm{~nm}$.

We have shown the existence of stable coupled conjugate pulses in fiber four-wave mixing. These waves can be generated by injection of a single pulse at the signal wavelength and permit time localization to be maintained in the ultrashort conjugate pulse.

The research of S. Wabnitz was supported by the Centre National de la Recherche Scientifique. That of J. M. Soto-Crespo was supported by the Comunidad de Madrid under contract 06T/039/96 and the Comisión Interministerial de Ciencia y Tecnología under contract TIC95-0563-03.

\section{References}

1. A. Yariv, D. Fekete, and D. Pepper, Opt. Lett. 4, 52 (1979).

2. S. Watanabe, T. Chikama, G. Ishikawa, T. Terahara, and H. Kuwahara, IEEE Photon. Technol. Lett. 4, 1241 (1993).

3. W. Forysiak and N. J. Doran, Electron. Lett. 30, 154 (1994).

4. K. Inoue, J. Lightwave Technol. 10, 1553 (1992).

5. S. Watanabe and T. Chikama, Electron. Lett. 30, 163 (1994).

6. S. Trillo, S. Wabnitz, E. M. Wright, and G. I. Stegeman, Opt. Lett. 13, 871 (1988).

7. G. P. Agrawal, Nonlinear Fiber Optics, 2nd ed. (Academic, New York, 1995).

8. K. Suzuki, M. Nakazawa, and H. A. Haus, Opt. Lett. 14, 320 (1989); P. Franco, F. Fontana, I. Cristiani, M. Zenobi, M. Midrio, and M. Romagnoli, Opt. Lett. 21, 788 (1996)

9. N. N. Akhmediev, V. I. Kornev, and Yu. V. Kuz'menko, Zh. Eksp. Teor. Fiz. 88, 107 (1985) [Sov. Phys. JETP 61, 62 (1985)].

10. J. M. Soto-Crespo, N. N. Akhmediev, V. V. Afanasjev, and S. Wabnitz, Phys. Rev. E 55, 4784 (1997). 